\title{
A THEORETICAL PERIOD-LUMINOSITY RELATION OF DWARF CEPHEIDS
}

\author{
D.H. McNamara and John M. Powell \\ Department of Physics and Astronomy \\ Brigham Young University \\ Provo, Utah 84602 USA
}

Dwarf Cepheids (large-amplitude $\delta$ Sct variables and SXPhe variables in the GCVS) are late A-type stars near light maximum and early-F stars near light minimum. In the lower part of the instability strip where these stars are found the pulsation constant $Q$ varies only slightly. As pointed out by Andreasen, Hejlesen, and Petersen (1983) this makes it easy to transform a theoretical HR or alternatively a $\left(\log T_{e}, \log g\right)$ diagram to a theoretical $\left(\log T_{e}, \log P_{0}\right)$ diagram by the use of the $P_{0} \sqrt{\rho}=Q$ relation. This follows from the equations:

$$
\begin{gathered}
\log P_{0}=\log Q+1.5 \log R / R_{\odot}-0.5 \log M / M_{\odot} \\
\log g=\log g_{\odot}+\log M / M_{\odot}-2 \log R / R_{\odot},
\end{gathered}
$$

where $P_{0}$ is the fundamental period of pulsation. The periods, of course, can be determined with very high accuracy.

For a given chemical composition $Z$, the evolutionary tracks of stellar models provide a direct means to calibrate $\left(\log T_{e}, \log P_{0}\right)$ as a function of mass. To this end we have utilized the models of both Mengel et al. (1979) and VandenBerg (1985) to produce evolutionary tracks in $\left(\log T_{e}, \log P_{0}\right)$ diagrams as a function of mass and chemical composition $Z$ for an assumed helium abundance, $Y=0.25$. The two sets of models give similar results. With the aid of the period and the effective temperature, $T_{e}$, the mass and also the bolometric magnitude can be inferred from the $\left(\log T_{e}, \log P_{0}\right)$ diagram and position of the star in the traditional HR diagram. We have utilized published photometry, primarily $u v b y \beta$ and Kurucz (1988), atmosphere models to estimate the mean effective temperature of the stars. Bolometric magnitudes of 25 dwarf Cepheids have been determined and the results are shown in Figure 1 where $M_{\text {bol }}$ is plotted as a function of $\log P_{0}$. The open squares represent our values as inferred from the periods and effective temperatures at mean light. The solid line is the period-luminosity relation given by the equation

$$
M_{\text {bol }}=-3.762 \log P_{0}-2.007
$$

based on a least-square solution.

We also have plotted as solid squares the bolometric magnitudes of 14 metalpoor variables with light amplitude $\geq 0 .^{m} 25$ found in globular star clusters (Nemec and Mateo 1990). 


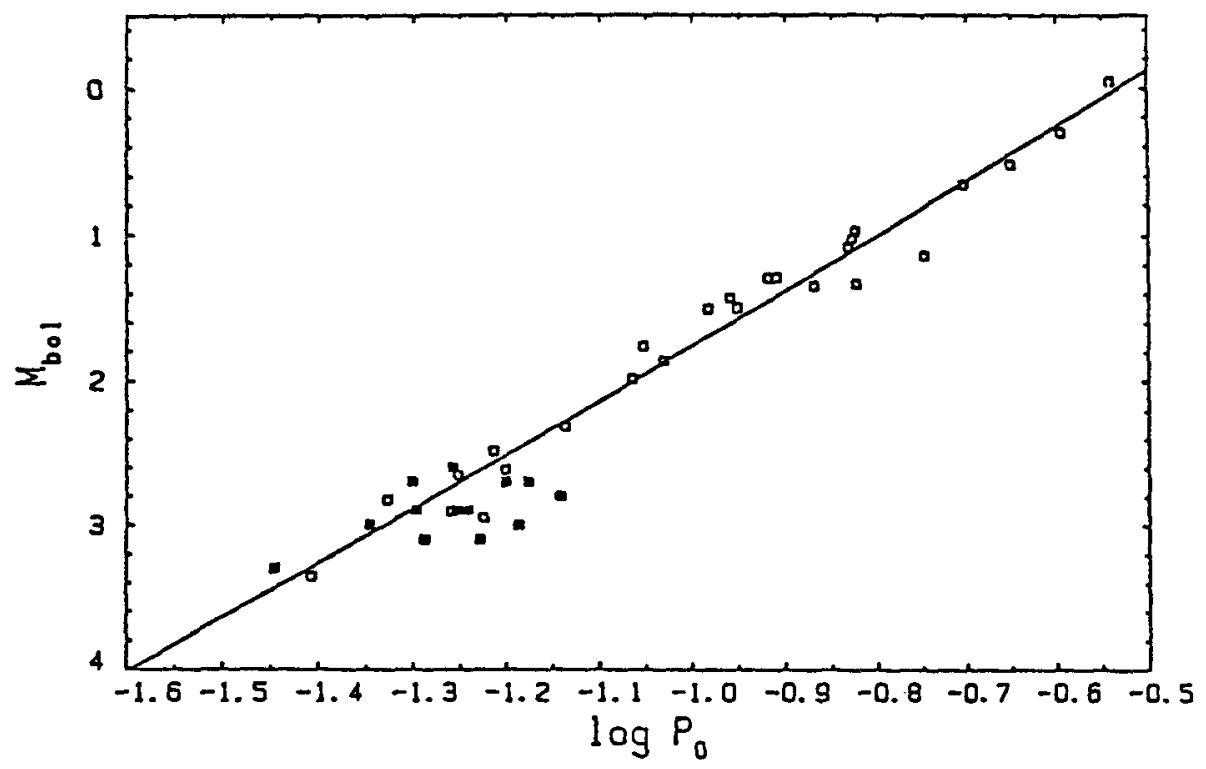

Fig. 1 - Theoretical period - luminosity relation of dwarf Cepheids. Open squares are $M_{\text {bol }}$ values derived from models and the $\left(P_{0}, T_{e}\right)$ values of the variables. The solid squares are $M_{\mathrm{bol}}$ values of metal-poor variables found in globular star clusters.

A bolometric correction of $-0 .{ }^{m} 2$ has been applied to the $M_{v}$ values given by Nemec and Mateo. The $M_{v}$ values were determined by fitting HR diagrams of the stars in the cluster to standard HR diagrams. Note that the observed $M_{\text {bol }}$ values are $\sim 0 .{ }^{m} 2$ fainter than the theoretical curve. The origin of this small discrepancy is unknown at the present time.

\section{References}

Andreasen, G.K., Hejlesen, P.M., and Petersen, J.O. 1983, A\&A, 121, 241.

Kurucz, R.L. 1988, unpublished.

Mengel, J.G., Sweigart, A.V., DeMarque, P., and Gross, P.G. 1979, APJS, $\underline{40}$, 733.

Nemec, J., and Mateo, M. 1990 in Confrontation Between Stellar Pulsation and Evolution, eds. C. Cacciari and G. Clementini, ASPCONF SER 11, 64.

VandenBerg, D.A., 1985, APJS, $\underline{58}, 711$. 